\title{
Geometric Conditions forStarlikenessand Convexity of Univalent Functions
}

\author{
${ }^{1}$ A.A. James, ${ }^{2}$ A. O. Lasode, ${ }^{3}$ B.O. Moses. \\ ${ }^{1}$ Mathematics Division, School of Arts and Sciences, American University of NigeriaYola, Adamawa \\ State, Nigeria. \\ ${ }^{2}$ Department of Mathematics, Faculty of Science, University of Ilorin, Ilorin, Kwara State Nigeria. \\ ${ }^{3}$ Department of Mathematics, College of Sciences, LandMark UniversityOmuaran, Kwara State, Nigeria.
}

\begin{abstract}
It is of interest in this work to analyze the sufficient conditions of Starlike and Convex functions using the geometric approach. The domains are preserved by the Conformal Mapping Principle.Furthermore, we established subordination properties for functions of the class $T_{n}^{\alpha}(\beta)$ using our new approach.
\end{abstract}

Keyword: Convexity, Starlikeness, Univalent, Unit disk,

\subsection{BACKGROUND TO THE STUDY}

\section{Introduction}

The study of analytic functions has received much attention of researchers over the last century. Due to its application in many physical problems like heat conduction, electrostatics potential, fluid flows and also its interdependence with many specialized areas of mathematical endeavours.Opoola T.O, [15] introduced a new class of univalent function in 1994. This class has received great attention by researchers such as Babalola, K.O and Opoola 2006, Oladipo, A.T. to mention few.

This section is focused on some basic and general definitions which are related to the scope of this research work. We also give few examples where necessary to lay a solid foundation for the main results which shall come later in the work.

\subsection{SOME BASIC DEFINITIONS \\ 1.2.1: UNIT DISK}

Let denote the unit disk by $E$. The set of all points $z \in E$ whose modulus are less than one; $E=\{z:|z|<1\}$ in the complex plane $C$ is called the unit disk.

\subsection{2: OPEN SETS}

An open set is a set which consists only of interior points. For example,the set of points $z$ such that $|z|<1$ is an open set.

\subsection{3: OPEN DOMAINS}

An open connected set in the complex plan is called an open domain.

\subsection{4: NEIGHBOURHOODS}

A $\delta$ neighborhood of a point $z_{0}$ is the set of all points $z$ such that $\left|z-z_{0}\right|<\delta$ where $\delta$ is any positive number.

\subsection{5: (A DELETED $\delta$ NEIGHBOURHOOD)}

A deleted $\delta$ neighborhood of $z_{0}$ is a neighborhood of $z_{0}$ in which the point $z_{0}$ is omitted that is $0<\left|z-z_{0}\right|<\delta$.

\subsection{6: (DERIVATIVES)}


If $f(z)$ is single-values in some domain $D$ of the $z$ plane, the derivative of $f(z)$ is defined as $f^{\prime}(z)=\lim _{\Delta Z \rightarrow 0} \frac{f(z+\Delta Z)-f(z)}{\Delta Z}$

Provided the limit exist as $\Delta Z \rightarrow 0$. In such case we say that $f(z)$ is differentiable at $z$.

\subsection{7: (ANALYTIC FUNCTION)}

If the derivative $f^{\prime}(z)$ exists at all points $z$ of a domain $D$, then $f(z)$ is said to be analytic in $D$ and is referred to as an analytic function in $D$ or a function analytic in $D$. The terms regular and holomorphic are sometimes used as synonyms for analytic.

A function $f(z)$ is said to be analytic at a point $z_{0}$ if there exists a neighborhood $\left|z-z_{0}\right|<\delta$, all points of which $f^{1}(z)$ exists.

\subsection{8: (CONFORMAL MAPPING)}

A conformal mapping is a transformation $w=f(z)$ that preserves angles between oriented curves in magnitude as well as in senses. An analytic function is conformal at any point where it has a nonzero derivative. Any conformal mapping of a complex variable which has a continuous partial derivative is analytic.

\subsection{9: (SUBORDINATE FUNCTION)}

Let $f(z)$ and $g(z)$ be analytic in E. The function $f(z)$ is said to be subordinate to $g(z)$ denoted by $f(z) \prec g(z)$ if there exists an analytic function $w \in W$ such that $f(z)=g(w(z))$ for $z \in E$.

\subsubsection{0: UNIVALENT FUNCTION}

A single-valued analytic function $f(z)$ defined in a domain $D \subset E$ is said to be univalent if it never takes the same value twice that is for $z_{1}, z_{2} \in D$ if $f_{1}(z)=f\left(z_{2}\right) \Rightarrow z_{1}=z_{2}$ and equivalently if $z_{1} \neq z_{2} \Rightarrow f(z) \neq f\left(z_{2}\right)$.

A function $f(z)$ is said to be univalent in a domain $D \subset E$ if it provides a one-to-one mapping onto its image.

An analytic and univalent function $f(z)$ in a unit disk is said to be normalized when $f(0)=0$ and $f^{\prime}(0)=1$.

Geometrically

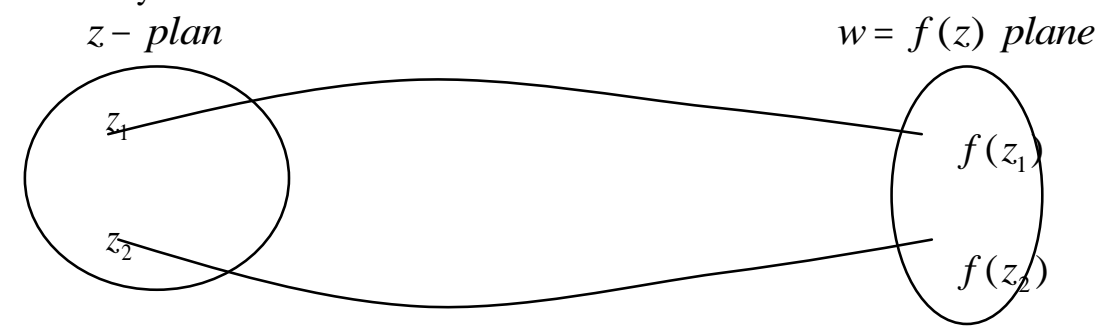

\subsubsection{1: NORMALIZED UNIVALENT FUNCTION}

If $g(z)$ is regular in $E$ then it has a Maclaurin expansion of the form.

$$
\begin{aligned}
& g(z)=b_{0}+b_{1} z+b_{2} z^{2}+\ldots \sum_{n=0}^{\infty} b_{n} z^{n} \text { is convergent in } E . \\
& \text { If } g(z)-b_{0}=\sum_{n=0}^{\infty} b_{n} z^{n}-b_{0}
\end{aligned}
$$


$\Rightarrow g(z)-b_{0}=\sum_{n=1}^{\infty} b_{n} z^{n}$

Let divide both sides by $b_{1}$

$\Rightarrow \frac{g(z)-b_{0}}{b_{1}}=\sum_{n=1}^{\infty} \frac{b_{n}}{b_{1}} z^{n}$

set $f(z)=\frac{g(z)-b_{0}}{b_{1}}$ and

$\frac{b_{n}}{b_{1}}=a_{n} \therefore$ since $g(z)$ is univalent in $D$ then $f(z)$ is also univalent Hence we arrive at

$f(z)=z+\sum_{n=2}^{\infty} a_{n} z^{n}$

$\Rightarrow f(z)=z+a_{2} z^{2}+a_{3} z^{3} \ldots+\sum_{n=2}^{\infty} a_{n} z^{n}$

Which is the normalized univalent function

\subsubsection{2: STARLIKE DOMAIN}

A domain $D$ is said to be starlike with respect to $w_{0}$ if $\forall w_{0} \in D$. The line $t w_{0}+(1-t) w \in D$. In the special case when $w_{0}=0$, we say that $D$ is starlike, for $t \in[0,1]$ or A domain $D \subset C$ is said to be starlike with respect to a point $w_{0} \in E$ if the line segment joining $w_{0}$ to every other point $w \in D$ lies entirely in $D$.

\subsubsection{4: CONVEX DOMAIN}

A domain $D$ is said to be convex if $\forall w_{1}, w_{2} \in D$, in the interior of $D$, the line segment $t w_{1}+(1-t) w_{2} \in D, t[0,1]$. In other words a set $D$ is said to be convex if it is starlike with respect to each of its points, that is, if the linear segment joining any two points of $D$ lies entirely in $D$.

\section{Main Results}

\subsection{DERIVATION OF SUFFICIENT CONDITIONS FOR CONVEXITY AND STARLIKENESS} Introduction:

The trigonometric form of the complex number $z(x, y)$ is given by $x=r \cos \theta$ and $y=r \sin \theta$ where $\mathrm{r}$ is the radius, $\theta$ is the argument and $\mathrm{z}$ is a complex number of two variables.

$$
\begin{aligned}
z=x+i y & =r \cos \theta+i \sin \theta \\
& =r(\cos \theta+i \sin \theta) \\
& =r e^{i \theta}
\end{aligned}
$$

Geometrically

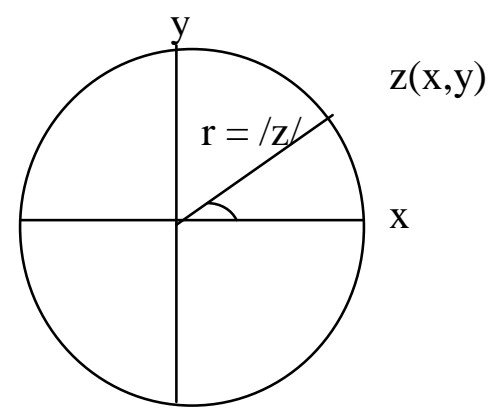

Hence $z=r e^{i \theta}$ is a boundary point representation. If $r=1$ then $z=e^{i \theta}$ 
Generally, equation of circle can be written as $|z-\varsigma|=r$ In polar form

$$
\begin{aligned}
& z-\varsigma=r e^{i \theta} \\
& \Rightarrow z=\varsigma+r e^{i \theta}
\end{aligned}
$$

\subsection{CONVEXITY SUFFICIENT CONDITION}

If $w=f(z)$

$$
f^{\prime}(z)=\lim _{z \rightarrow \Delta z} \frac{f(z+\Delta z)-f(z)}{\Delta z}=\frac{\Delta w}{\Delta z}
$$

Geometrically,
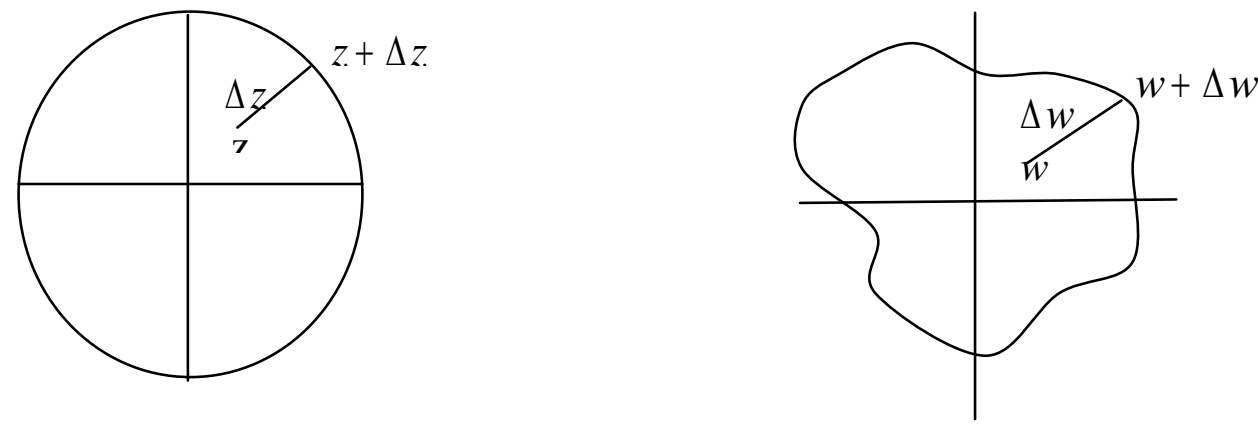

i.e. $\quad \Delta w=f(\Delta z)$

now consider

let $\sigma=\{z:|\varsigma|=\delta\}$

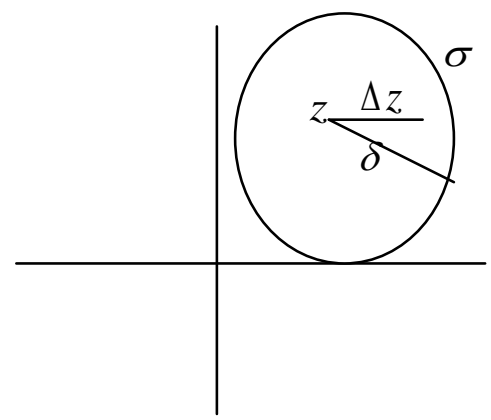

Let $\Delta z \in \sigma,|\Delta z|<\delta$

Let $\Delta z \in \varsigma e^{i \theta}, \varsigma>0$

But

$$
\frac{\Delta w}{\Delta z}=f^{\prime}(z)+\alpha \Delta z
$$

$$
\alpha(\Delta z) \rightarrow 0 \text { as } \Delta z \rightarrow 0
$$

Now $\Delta z=f^{\prime}(z) \Delta z$

$$
f^{\prime}(z)=r e^{i \theta}
$$

Let $\Delta w=r e^{i \varphi} \varsigma e^{i \theta}$

$$
=r \varsigma e^{i \theta}(\varphi+\theta)
$$


$|\Delta w|=r \varsigma \quad$ since $\left|e^{(i \varphi+\theta)}\right|=1$

Geometrically

$$
\theta_{2}-\theta_{1}
$$
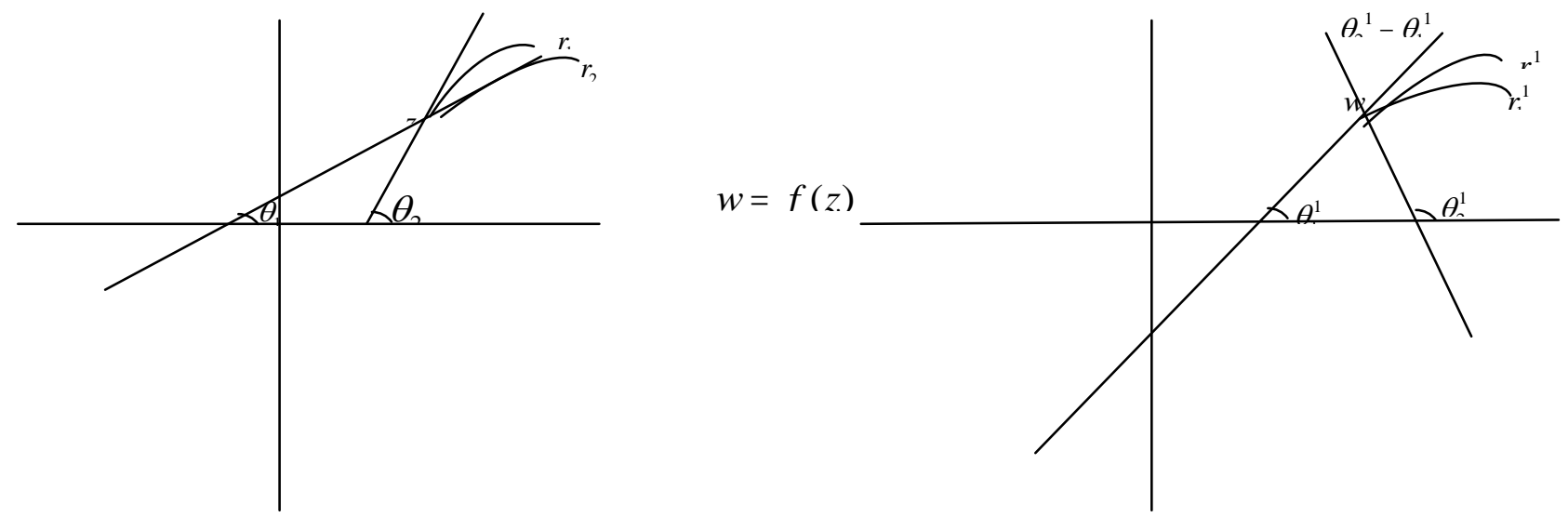

But argument $\Delta w=\varphi+\theta$

$$
\operatorname{Arg} \Delta w=\arg f^{\prime}(z)+\arg \Delta w
$$

Now with

$$
z=r e^{i \theta}
$$

\section{Remark}

Let

$$
\begin{aligned}
& \theta_{1}^{1}=\theta_{1}+\varphi \\
& \theta_{2}^{1}=\theta_{2}+\varphi
\end{aligned}
$$

Subtract 2.1 from 2.2 gives

$$
\begin{aligned}
& \theta_{1}^{1}-\theta_{2}=\theta_{1}-\theta_{2} \\
& \theta_{1}^{1}=\theta_{1}+\theta_{2}^{1}-\theta_{2} \text { also }
\end{aligned}
$$

Subtract 2.2.2 from 2.2.1 gives

$\theta_{2}^{1}-\theta_{1}^{1}=\theta_{2}-\theta_{1}$

$\theta_{2}^{1}-\theta_{1}^{1}>0$ if and only if

$$
\theta_{2}-\theta_{1}>0
$$

$\Rightarrow \theta_{2}>\theta_{1}$ and

$\theta_{2}^{1}-\theta_{1}^{1}>0$ if and only if $\theta_{2}>\theta_{1}$,

Also $\theta_{2}^{1}-\theta_{1}^{1}<0$ if and only if

$$
\begin{aligned}
& \theta_{2}-\theta_{1}<0 \\
& \Rightarrow \theta_{2}<\theta_{1} \text { and } \\
& \theta_{2}^{1}-\theta_{1}<0 \text { if and only if } \\
& \theta_{2}<\theta_{1}
\end{aligned}
$$

By conformal mapping angles are preserved. That is the angle between any two smooth curves intersecting at the point $z_{0}$ is equal in magnitude and sense to the angle between their images in the $w$-plane at the point $w_{0}=f\left(z_{0}\right)$. 
Geometrically

Hence to show that $\frac{\partial \psi}{\partial \varphi} \geq 0$

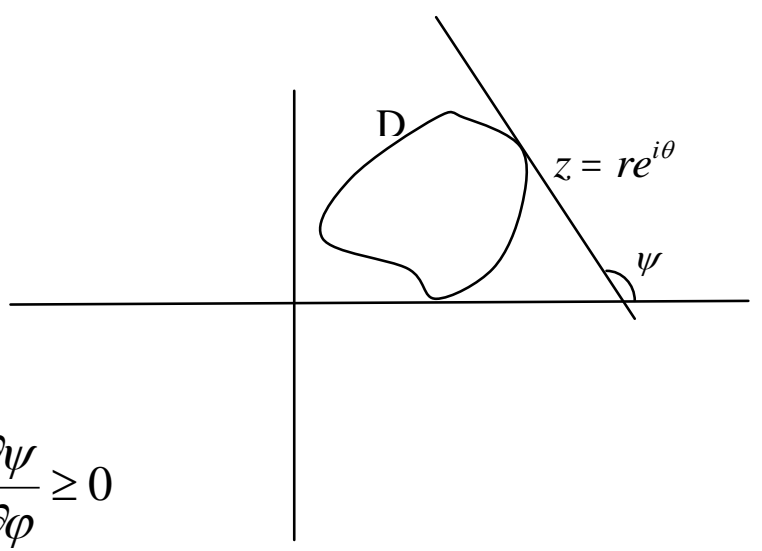

$\Rightarrow \operatorname{Re}\left[1+\frac{z f^{\prime \prime}(z)}{f^{\prime}(z)}\right]>0$

From (4.1)we have

$$
\Delta w=\arg \Delta w \arg f^{\prime}(z)
$$

Also we know tha

$|z-\varsigma|=r$

$z-\varsigma=r e^{i \varphi}$
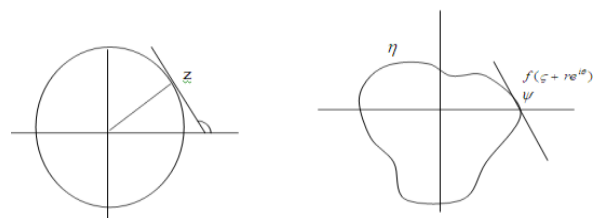

$z=\varsigma+r e^{i \varphi}$

By maximum modulus theorem, the maximum paint of $|f|$ is on the boundary, hence the domain above is convex if and only if $\frac{\partial \psi}{\partial \varphi} \geq 0$

Now by the fact that

$\Delta w=\arg \Delta z+\arg f^{\prime}(z)$

let $\varphi=\arg \left(\varsigma+r e^{i \varphi}\right)+\arg f^{\prime}\left(\varsigma+r e^{i \varphi}\right)$

so $\varphi=\varphi+\pi / 2+\arg f^{\prime}\left(\varsigma+r e^{i \varphi}\right)$

But

$\operatorname{Im} \int_{\eta} \frac{f^{\prime \prime}(\varsigma)}{f^{\prime}} d \varsigma=\int_{\eta} d \ln f^{\prime}=\operatorname{lm}\left(\ln f^{\prime}(z)\right)$

$=\operatorname{Im}\left(\ln \left|f^{\prime}\right|+i \arg f^{\prime}(z)\right)$

$=\arg f^{1}$

So substituting into equation (4.4) gives 
$\psi=\varphi+\pi / 2+\operatorname{lm} \int_{\eta} \frac{f^{\prime \prime}(\varsigma)}{f^{\prime}(\varsigma)} d \varsigma$

Hence

$$
\begin{aligned}
& \frac{\partial \psi}{\partial \varphi}=\frac{\partial}{\partial \varphi}\left(\varphi+\pi / 2+\operatorname{lm} \int_{n} \frac{f^{\prime \prime}(\varsigma)}{f^{\prime}(\varsigma)} d \varsigma\right) \\
& =1+0+\frac{\partial}{\partial \varphi}\left(\operatorname{lm} \int \frac{f^{\prime \prime}(\varsigma)}{f^{\prime}(\varsigma)} d \varsigma\right) \\
& \frac{\partial \psi}{\partial \varphi}=1+\frac{\partial}{\partial \varphi}\left(\operatorname{lm} \int_{0}^{2 \pi} \frac{f^{\prime \prime}(\varsigma)}{f^{\prime}(\varsigma)} d \varsigma\right) \\
& =\operatorname{Re}\left(1+\frac{z f^{\prime \prime}}{f^{\prime}}\right)>0
\end{aligned}
$$

Hence it is convex

\subsection{STARLIKE SUFFICIENT CONDITION}

The sufficient condition of a starlike function is given as $\operatorname{Re}\left(\frac{z f^{\prime}(z)}{f(z)}\right)>0$

Proof

Let $w=f(z)$

That is $\Delta w=f(\Delta z)$

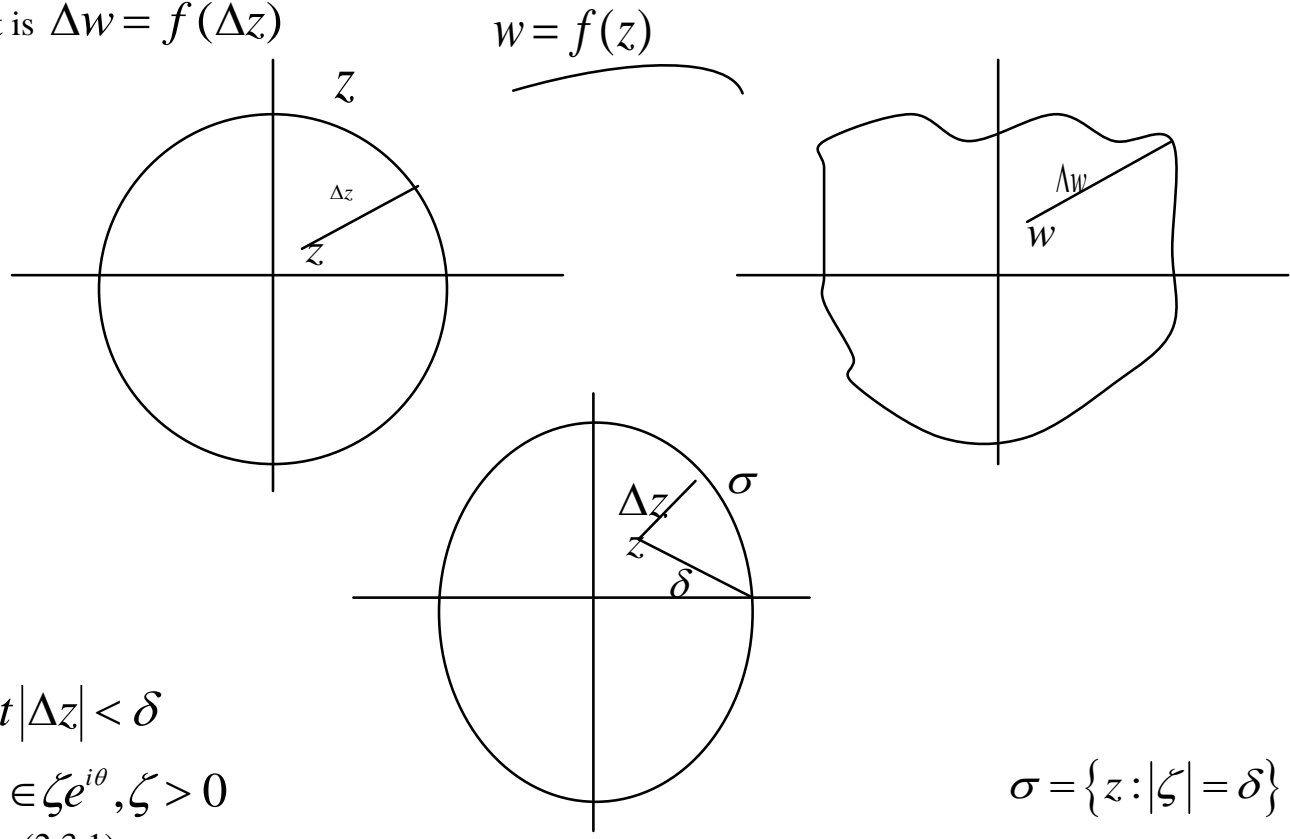

From (2.3.1) 
$\Delta w=f(\Delta z)$

let $f(\Delta z)=f\left(\zeta e^{i \theta}\right)$

$$
\begin{aligned}
& (\Delta w)=f\left(\zeta e^{i \theta}\right) \\
& |\Delta w|=\left|f\left(\zeta e^{i \theta}\right)\right| \\
& |\Delta w|=\zeta, \text { since }\left|e^{i \theta}\right|=1
\end{aligned}
$$

But $\arg \arg \Delta w=\arg f(\Delta z)$

It is sufficient to show that

$$
\frac{\partial \psi}{\partial \varphi} \geq 0 \Rightarrow \operatorname{Re}\left\{\frac{z f^{\prime}(z)}{f(z)}\right\}>0 \text { is Starlike }
$$

Geometrically
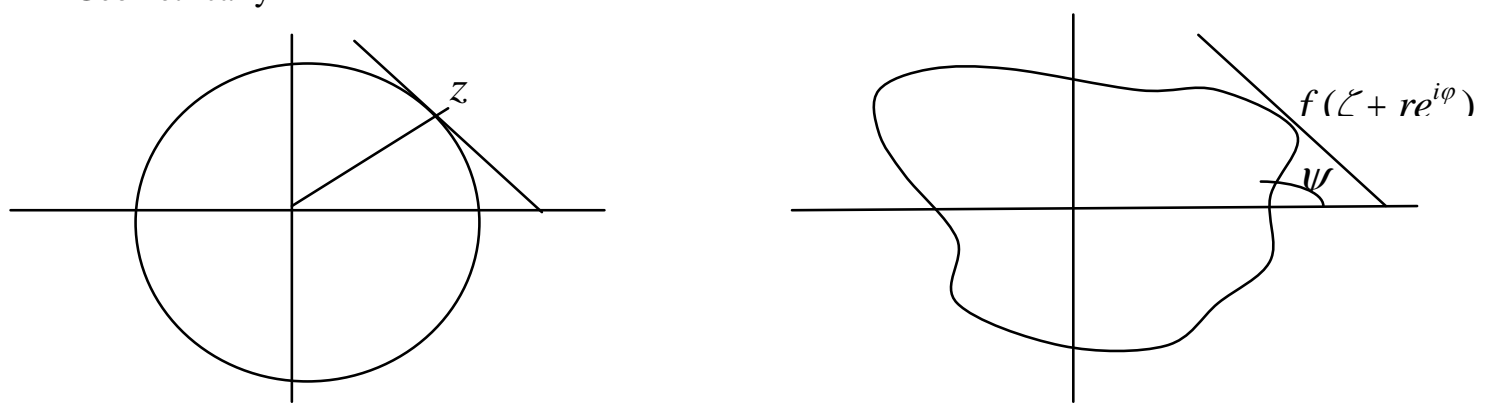

Note that by maximum modulus theorem and conformal mapping angles of the domains are preserved But

$$
\begin{aligned}
& |z-\zeta|=r \\
& z-\zeta=r e^{i \varphi} \\
& z=\zeta+r e^{i \varphi} \\
& \zeta=r e^{i \varphi}-z
\end{aligned}
$$

Also

$$
\begin{aligned}
& \operatorname{lm} \int_{\eta} \frac{f^{\prime}(\zeta)}{f(\zeta)} d \zeta \\
& =\operatorname{lm} \int_{\eta} d \ln f=\operatorname{lm}(\ln f) \\
& =\operatorname{lm}(\ln |f|+i \arg f) \\
& =\arg f(z)
\end{aligned}
$$

From 2.3.2

$$
\begin{aligned}
& \arg \Delta w=\arg +(\Delta z) \\
& \text { Let } \psi=\arg f(z) \\
& \psi=\operatorname{lm} \int_{\eta} \frac{f^{\prime}(\zeta)}{f(\zeta)} d \zeta \\
& \frac{\partial \psi}{\partial \varphi}=\frac{\partial}{\partial \varphi}\left(\operatorname{lm} \int_{\eta} \frac{f^{\prime}(\zeta)}{f(\zeta)}\right)
\end{aligned}
$$




$$
\begin{aligned}
& \Rightarrow \frac{d \psi}{d \varphi}=\operatorname{Im} \frac{d}{d \varphi} \int_{0}^{2 \pi} \frac{f^{\prime}\left(\zeta+r e^{i \theta}\right)}{f\left(\zeta+r e^{i \theta}\right)} i r e^{i \varphi} \\
& =\operatorname{Im} \frac{f^{\prime}\left(\zeta+r e^{i \theta}\right)}{f\left(\zeta+r e^{i \theta}\right)} i r e^{i \varphi} \\
& =\operatorname{Re} \frac{z f^{\prime}(z)}{f(z)}>0 \text { which is Starlike since } \frac{\partial \psi}{\partial \varphi}>0
\end{aligned}
$$

\section{Conclusion}

Using the geometrical approach to derive the sufficient conditions of both

Starlike functions $\operatorname{Re}\left\{\frac{z f^{\prime}(z)}{f(z)}\right\}>0$ and convex functions $\operatorname{Re}\left\{\frac{z+f^{\prime \prime}(z)}{f^{\prime}(z)}\right\}>0$.

The study of Salagean Differential Operator $D^{n} f(z)=D\left[D^{n-1} f(z)\right]$, and Ruscheweyh derivative $D^{n} f(z)=\frac{z}{(1-z)^{n+1}} f(z)$ gives an interesting result when $n=0,1,2$. These operators can be investigated further in future research work.

\section{References}

[1] Alexander, J.W. (1915). Function which map the interior of the unit circle upon simple regions.Annals of Mathematics. (17), 12-22.

[2] Babalola, K.O. (2005). Some new results on a certain family of analytic functions defined by theSalageanderivative.Ph.DThesis.Department of Mathematics, University of Ilorin.

[3] Babalola, K.O. and Opoola T.O, Iterated Integral transformsofCaratheodory function and their applications to analytic and univalent functions. Tamkang J. Math., 37(4)(2006), 355-366

[4] Bernard, S.D. (1966). Bibliography of schlichtfunctions.Courant Institute of Mathematical Science.New York. (Reprinted by Mariner Publishing Co. Inc. Tampa, Florida).

[5] Bernard, S.D. (1969). Convex and Starlikefunctions.Transactions of the American Mathematical Society (135) $429-446$.

[6] Caratheodory, C. (1960). Theory of functions of a complex variable. Vol. II Chelsea Publishing Co. New York.

[7] Dorff, M and Szynal, J. (2002). Higher order Schwarzian derivatives for convex univalent functions. Reprint submitted to Elsevier Science.

[8] Duren, P.L. (1977). Coefficients of univalent functions.Bulletin of the American Mathematical Society.(83), 891-911.

[9] Duren, P.L. (1983). Univalent functions. Springer-Verlag, New York.

[10] Goodman, A.W. (1983). Univalent functions. Mariner Publishing Co. Inc. Tampa, Florida.

[11] Hardy, G.H. (1952). A course in Pure Mathematics.Cambridge University Press, $10^{\text {th }}$ Edition.

[12] Hardy, G.H., Litlewood, J.E. and Polya, G. (1934). Inequalities.Cambridge University Press p. 184.

[13] Hayman, W.K. (1958). Multivalent functions. Cambridge Tracts in Mathematics and Mathematics - Physics.Cambridge University Press.p 48

[14] Hille, E. (1962). Analytic function theory.Vol. II Chelsea Publishing Co. New York.

[15] T.O., Opoola, On a new subclass of univalent function; Mathematika Tome(36) 59 No2 (1994), 195-200

[16] Noonan, J.W. (1973). On close-to-convex of order $\beta$.Pacific Journal of Mathematics. (44) 263-280. 